\title{
Análisis bibliométrico de los trabajos de titulación de ocho universidades de Pichincha, Napo y Orellana (Ecuador)
}

\section{Bibliometric analysis of thesis from eight universities of Pichincha, Napo and Orellana (Ecuador)}

\author{
Nancy Estrella ${ }^{1, *}$, Xavier Lastra-Bravo ${ }^{1, *}$ \\ $凶$ Autor de correspondencia: nancy.estrella.4a@gmail.com,xblastra@uce.edu.ec \\ 1. Universidad Central del Ecuador. Facultad de Ciencias Agrícolas. Carrera de Turismo Ecológico. Jerónimo Leiton y Av. La Gasca s/n. Ciudadela \\ Universitaria. Quito. 170521. Ecuador. \\ Artículo original de la ponencia presentada en el IV CONGRETUR - "El impacto del turismo en el desarrollo del Ecuador", Universidad Central \\ del Ecuador, 21 al 24 de noviembre de 2018
}

\section{Resumen}

El análisis bibliométrico es útil para analizar la cantidad y la calidad de las publicaciones científicotécnicas, como las temáticas y áreas de estudio. Las universidades generan producción científico-técnica a través de los trabajos de titulación que desarrollan sus estudiantes, pero no han recibido el mismo tratamiento de otras formas de divulgación como artículos científicos o ponencias a congresos. En este sentido, en Ecuador no se han realizado análisis bibliométricos de los trabajos de titulación sobre turismo desarrollados en sus universi-dades. Por ello, se planteó como objetivo sistematizar los trabajos de titulación de pregrado y posgrado de ocho universidades de las provincias de Pichincha, Napo y Orellana, que poseen carreras relacionadas con turismo, hospitalidad y gastronomía, en el período 2000-2017. Se sistematizó la información disponible en los reposito-rios digitales, se aplicó estadística descriptiva y se calcularon índices bibliométricos. Como resultado se reco-pilaron 3.185 trabajos de titulación, principalmente de pregrado (98\%). Se identificó que el 62\% se enfocan en temas económicos y administrativos, orientados al sector privado, concentrados en Pichincha (especialmente en el Centro Histórico y La Mariscal), Cotopaxi e Imbabura. Otras temáticas tratadas son turismo comunitario y cultural, principalmente en la Sierra y Amazonía, y desarrollo turístico de los territorios. Se identificaron las universidades con mayor productividad y grado de colaboración y, con los grupos de investigación más grandes. La literatura citada tiene un envejecimiento alto, principalmente de México y España. Los resultados obtenidos pueden ser un insumo importante para los sectores público, privado y comunitario, y en especial la academia, que podría enfocar futuras investigaciones en temáticas y zonas poco estudiadas.

Palabras clave: Análisis Bibliométrico, Investigación en Turismo, Índices Bibliométricos, Producción académica, Ecuador

\begin{abstract}
Bibliometric analysis is useful for quantitative and qualitative analysis of scientific-technical publications, as well as establishing topics and research areas. Universities produce scientific and technical knowledge through degree projects developed by their students, but it has not received the same treatment as other dissemination forms such as scientific papers or conference presentations. In this sense, a bibliometric analysis of degree projects on tourism from Universities of Ecuador has not been done. Hence, this research states as objective the systematization of undergraduate and graduate degree projects from eight Universities of Pichincha,
\end{abstract}


Napo, and Orellana provinces, which have programs on tourism, hospitality, and gastronomy. The available information in digital repositories was systematized from the period of 2000 to 2017. Descriptive statistics and bibliometric indexes were applied. As a result, 3.185-degree projects were collected, most of them (98\%) were from undergraduate programs. $62 \%$ of degree projects were identified and focused on economic and administrative topics based on the private sector mainly in Pichincha (especially in Quito's Downtown and La Mariscal neighborhoods), Cotopaxi and Imbabura provinces. Community-based and cultural tourism topics, mainly in Highlands and Amazonia, and tourism development for the territories were also addressed. Universities with greater productivity, levels of collaboration and bigger research teams were identified. The literature cited is not up-to-date and it comes mainly from Mexico and Spain. The results obtained can be an important input for public, private, and community sectors and mainly for Academia, which could focus its further researches on topics and least studied areas.

Keywords: Bibliometric analysis; Research on Tourism; Bibliometric Index; Academic production; Ecuador

\section{Introducción}

El estudio de los documentos escritos se remonta hacia la época de los griegos, quienes analizaban sus manuscritos con el fin de determinar los costos y remuneraciones, a este proceso se lo denominó "esticometría" (Camps, 2008). En la edad moderna el primer trabajo bibliométrico fue desarrollado por Alphonse de Consolle en 1885, quien aplicó métodos matemáticos para el análisis comparativo de las publicaciones científicas europeas y estadounidenses (Rueda-Clausen Gómez, Villa-Roel Gutiérrez, \& Rueda-Clausen Pinzón, 2010). En este sentido, a la bibliometría antiguamente se la entendía como la disciplina cuyo objeto de estudio eran los libros, este enfoque era muy limitado y se centraba únicamente en una temática particular y estaba asociada a la gestión bibliotecaria (Camps, 2008). Posteriormente, en los años setenta y ochenta, la bibliometría tomó un giro multidisciplinar y se la empezó a utilizar como un método de análisis y evaluación de la literatura (Peralta González, Frías Guzmán, \& Chaviano, 2015).

La bibliometría es una herramienta funcional para la evaluación de la literatura científica, basada en la codificación numérica de las características bibliográficas o formas de expresión de la comunicación escrita y la aplicación de métodos matemáticos y estadísticos a estos códigos, de manera que se evalúe la naturaleza y el curso de la disciplina sobre la que verse la bibliografía considerada (Amat Noguera, 1994; Broadus, 1987; Ferreiro Aláez, 1993; Pritchard, 1969). En relación con las definiciones dadas por los autores anteriormente mencionados, se entendería que la bibliometría es un estudio cuantitativo que se realiza a la literatura, ya sea esta libros, revistas, artículos científicos o tesis, y sirve para determinar la actividad científica que han tenido tanto los autores como las instituciones que los publican y de igual manera el impacto que ha generado la información para nuevas publicaciones. Más aún cuan- do la producción científica es la línea final de todas las actividades académicas y la investigación es el instrumento mediante el cual la comunidad científica muestra los resultados y presenta su trabajo al exterior (Bienert, Oliveira, Andrade, \& Caramori, 2015). El resultado de la búsqueda debe ser creíble, ser accesible y, después de publicado, no debe modificarse. Además, deben identificarse claramente los diferentes análisis de la base de datos de investigación primaria.

Por ello, en la actualidad, la bibliometría se la usa con más frecuencia, y en diferentes disciplinas y áreas temáticas, y ayuda a valorar la literatura científica de un país en relación con el resto del mundo, de una institución en relación con su país y a los autores en relación con su comunidad, y cómo esta utiliza dicha información (Peralta González et al., 2015). Más aún cuando la revisión de la literatura científica es un elemento importante a la hora de elaborar nuevos proyectos, nuevas investigaciones o nuevas publicaciones.

Para llevar a cabo estudios bibliométricos, se requiere información de calidad y actualizada sobre los temas de interés que se desea tratar, y en especial que su difusión ayude a satisfacer las necesidades sociales existentes (Solano López, Castellanos Quintero, López Rodríguez del Rey, \& Hernández Fernández, 2009). Los objetivos más importantes de esta herramienta son: el estudio del tamaño, crecimiento y distribución de los documentos al igual que la dinámica de los grupos que la producen y consumen dicha literatura y la información que contiene (González de Dios, Moya Benavent, \& Mateos Hernández, 1997). Entre las principales características del análisis bibliométrico se pueden destacar, su aplicación a diferentes áreas temáticas de investigación científica; la evaluación de las tendencias del conocimiento mediante técnicas cuantitativas, y el análisis que se aplica es descriptivo y retrospectivo (Pineda Ospina, 2015).

En Ecuador la bibliometría ha sido utilizada principalmente como herramienta para el desarro- 
1lo de trabajos de titulación y artículos científicos, entre algunos de los trabajos se citan "Indicadores bibliométricos de las tesis de pregrado en el repositorio digital de la Facultad de Ciencias Humanísticas y Sociales de la Universidad Técnica de Manabí en el período 2015-2016" (Díaz Macías \& Rodríguez Cevallos, 2017), “Análisis bibliométrico de la literatura científica publicada en la revista La Técnica (2010-2016)" (Torrijo Quindemil \& Cedeño Espinoza, 2017), "Análisis bibliométrico de la producción científica en ciencias de la salud en Ecuador (1999-2009), Hacia una política de investigación y desarrollo en función de las prioridades de salud del país" (Sisa Caiza, 2010), "Reflexiones sobre las potencialidades y límites de los índices de Cienciometría (bibliometría científica) convencionales (2015)" (Breilh, 2015), y "Análisis bibliométrico de la producción científica en el sistema de educación superior ecuatoriano" (Morocho Guerra, 2018).

Las áreas temáticas analizadas mediante la bibliometría aún son muy limitadas en Ecuador, igualmente no todas las universidades tienen en sus repositorios digitales temas relacionados a este. Por ende, es necesario seguir evaluando a la literatura científica en muchos más campos del conocimiento, tanto en temas de ciencias exactas como de ciencias sociales. La realización de estos estudios es importante porque ayuda a conocer la realidad de la actividad científica, como se maneja, la evolución con el paso del tiempo y para comparar el impacto que ha generado la ciencia en un área temática determinada.

En relación con la disciplina objeto de estudio en este artículo, el sector turístico es una actividad económica de gran importancia en distintos países y regiones, que relaciona la sociedad, la cultura, la naturaleza, el deporte y el tiempo libre (Moragues Cortada, 2006). Por ello en la actualidad, distintas instituciones gubernamentales, académicas y empresariales, se han enfocado en realizar estudios y publicaciones científicas sobre el turismo, de distinto formato como libros, artículos científicos, revistas, tesis, etc. Así como también, es importante considerar que existe un deseo generalizado entre los tomadores de decisiones de calificar y cuantificar las investigaciones realizadas (Ellegaard \& Wallin, 2015), siendo el turismo un campo en el que también se requiere este tipo de análisis.

A nivel internacional se han llevado a cabo distintos estudios bibliométricos en turismo, entre ellos "El ocio y el turismo en los artículos publicados en revistas académicas de turismo" (Azevedo, Faria, Gomes, Federal, \& Gerais, 2013), "Investigación en turismo activo: Revisión bibliográfica (1975-2013)" (Durán-Sánchez, Álvarez-García, \& Del Río-Rama, 2015),
"Convergence in tourism management research: a bibliometric analysis" (Estevão, Garcia, \& Fernandes, 2017), "Bibliometric Analysis of Tourism Research for the Period 2007-2016" (Güzeller \& Çeliker, 2018), "Bibliometric analysis of tourism and hospitality related articles published in Turkey" (Evren \& Kozak, 2014), "Bibliometric analysis of publications on wine tourism in the databases Scopus and WoS" (Durán-Sánchez, Del Río-Rama, \& Álvarez-García, 2017), "Experimental Researches in Tourism - A Bibliometric Analysis" (Güzeller \& Çeliker, 2018), "Trends and patterns in sustainable tourism research: a 25-year bibliometric analysis" (Ruhanen, Weiler, Moyle, \& Mclennan, 2015), "The Use of New Data Analysis Techniques in Tourism: A Bibliometric Analysis in Data Mining, Big Data and Structural Equations Models" (Palomo, Figueroa-Domecq, Flecha-Barrio, \& Segovia-Pérez, 2017), "Tourism and Sustainability: A Bibliometric and Visualization Analysis" (Tintoré Subirana, Diedrich, Vizoso, \& Pitarch, 2007)(Garrigos-Simon et al., 2018), "A bibliometric study of psychological research on tourism" (Barrios, Borrego, Vilaginés, Ollé, \& Somoza, 2008), "A Tri-Method Approach to a Review of Adventure Tourism Literature: Bibliometric Analysis, Content Analysis, and a Quantitative Systematic Literature Review" (Cheng, Edwards, Darcy, \& Redfern, 2018), "TOURISMOS: A Bibliometric Study" (Satyanarayana, 2014). Pero en Ecuador no se ha llevado a cabo un trabajo en este sentido.

Por la importancia creciente de las investigaciones en turismo y la utilidad de los análisis bibliométricos, se desarrolló el presente trabajo de investigación enfocado en analizar la producción académica generada a través de los trabajos de titulación, o comúnmente denominados "tesis", que desarrollan los estudiantes bajo la dirección de docentes tutores en las instituciones de educación superior (IES), que de acuerdo con la normativa ecuatoriana corresponden a universidades, escuelas politécnicas, institutos y conservatorios superiores. Los trabajos de titulación pueden ser desarrollados por máximo tres estudiantes y su financiación puede ser tanto pública como privada o autofinanciada.

Los trabajos de titulación de acuerdo con el Reglamento de Régimen Académico (Art. 21) corresponden a "proyectos de investigación, proyectos integradores, ensayos o artículos académicos, etnografías, sistematización de experiencias prácticas de investigación y/o intervención, análisis de casos, estudios comparados, propuestas metodológicas, propuestas tecnológicas, productos o presentaciones artísticas, dispositivos tecnológicos, modelos de negocios, emprendimientos, proyectos técnicos, trabajos experimentales, entre otros de similar nivel de 
complejidad" (Consejo de Educación Superior (CES), 2017). Los trabajos de titulación "deberán consistir en una propuesta innovadora que contenga, como mínimo, una investigación exploratoria y diagnóstica, base conceptual, conclusiones y fuentes de consulta". De acuerdo con el Código Orgánico de la Economía Social de los Conocimientos, Creatividad, e Innovación, los derechos de autoría de los trabajos de titulación corresponden a los estudiantes que lo desarrollan (Asamblea Nacional del Ecuador, 2016).

En este sentido, los trabajos de titulación cumplen un rol importante dentro del sistema educativo superior, ya que permite a los estudiantes adquirir capacidades investigativas, profundizar sus conocimientos en un campo específico de aplicación de su disciplina y cumplir con su requisito para titularse. Además, es una forma de que las IES en el Ecuador desarrollen proyectos investigativos a través de estudiantes y docentes tutores.

En esta investigación se sistematizaron todos los trabajos de titulación de pregrado y posgrado de ocho IES de Pichincha, Napo y Orellana, que corresponde a la Zona 2 establecida por la Secretaría Nacional de Planificación y Desarrollo (SENPLADES). En el caso de Pichincha se incluyeron las IES del Distrito Metropolitano de Quito (Zona 9). Las IES consideradas fueron Universidad Tecnológica Israel (U-ISRAEL), Universidad de las Fuerzas Armadas-ESPE (ESPE), Universidad San Francisco de Quito (USFQ), Universidad de Especialidades Turísticas (UDET), Universidad Tecnológica Equinoccial (UTE), Universidad Técnica Particular de Loja (UTPL), Universidad Iberoamericana del Ecuador (UNIBE), y Escuela Superior Politécnica Ecológica Amazónica (ESPEA). La información recolectada en esta investigación permitió determinar la cantidad y la calidad de la información publicada, conocer cómo se encuentra y cómo se maneja la actividad científica en cada institución, las áreas temáticas más tratadas o si existen ausencia de investigaciones en relación con determinados aspectos del turismo o zonas geográficas, también el ámbito geográfico en el que se han realizado los trabajos de titulación, y finalmente el cálculo de los índices bibliométricos relacionados con la productividad científica, el grado de colaboración entre autores, el nivel de antigüedad $u$ obsolescencia de la literatura citada y el nivel de apertura hacia investigaciones de otros países. Además, se utilizó la estadística descriptiva para caracterizar los trabajos de titulación.

\section{Materiales y métodos}

En la primera fase de la investigación se recopilaron los datos de los trabajos de titulación desarro- llados por los estudiantes y publicados en el período 2000-2017 en cada uno de los repositorios digitales de las ocho IES seleccionadas, y que están disponibles en las páginas web de cada una de ellas. Producto de la recolección de datos de los trabajos de titulación se obtuvo un total de 3.185 documentos, tanto de pregrado como de posgrado (Tabla 1), cuya información fue procesada en una base de datos. En el caso de la UNI$\mathrm{BE}$, solo se identificaron dos trabajos de titulación de gastronomía, los cuales no tenían disponible el documento para el respectivo análisis, por ello no fueron incluidos en la base de datos. La ESPEA, al ser una universidad cerrada, ya no tiene disponible su repositorio, por lo que no se pudo acceder para descargar los archivos correspondientes. Por ello, estas dos IES no fueron consideradas en el análisis.

Tabla 1. Número de publicaciones por IES

\begin{tabular}{ccc} 
IES & Trabajos de titulación & $\mathbf{\%}$ \\
\hline UTE & 2.036 & 63,92 \\
UDET & 372 & 11,68 \\
UTPL & 303 & 9,51 \\
ESPE & 183 & 5,75 \\
USFQ & 156 & 4,90 \\
U-ISRAEL & 133 & 4,18 \\
UNIBE & 2 & 0,06 \\
ESPEA & 0 & 0,00 \\
\hline TOTAL & $\mathbf{3 . 1 8 5}$ & $\mathbf{1 0 0 , 0 0}$ \\
\hline
\end{tabular}

En la base de datos se registró información sobre: universidad, carrera, modalidad de estudio, nivel (pregrado o posgrado), año de publicación, nombre del autor(es) y tutor(es) de tesis, tema, aplicación externa o interna a la IES (interna cuando se desarrolló el trabajo de titulación dentro de la misma IES), destinatario, ámbito geográfico de estudio, número de referencias bibliográficas y tamaño de grupo de investigación. En la variable autores y tutores, se analizó la dirección o tutoría de los trabajos de titulación, considerando que los autores (estudiantes) realizan únicamente un trabajo de titulación de pregrado o de posgrado. En la variable destinatario se consideraron proyectos públicos (desarrollados en conjunto o dirigidos a la administración pública en sus distintos niveles) o privados (desarrollados en conjunto o dirigidos a empresas particulares), en ámbito geográfico de estudio se establecieron cuatro niveles de aplicación (provincial, cantonal, parroquial, local), entendiéndose como local cuando el trabajo de titulación se aplicó a una empresa, entidad pú- 
blica o comunitaria concreta; parroquial cuando se estudió todo el territorio de la parroquia; cantonal y provincial cuando se consideró todo el territorio del cantón y provincia respectivamente. A partir de la información del ámbito geográfico de estudio se elaboraron mapas en ArcGIS para representar la densidad de trabajos por parroquias.

Los trabajos de titulación también se clasificaron de acuerdo con las líneas de investigación establecidas por la carrera de Turismo Ecológico de la Universidad Central del Ecuador: a) estudios económicos y administrativos, b) territorio, c) turismo alternativo $\mathrm{y}$ d) patrimonio turístico. Para clasificar los trabajos de titulación en dichas líneas de investigación se analizaron títulos, resúmenes y palabras claves, identificando las palabras claves utilizadas y la similitud de contenidos. Además, se clasificaron en sublíneas de investigación, considerando la creación de una sublínea con un mínimo de diez trabajos de titulación.

Para procesar los datos se utilizó la bibliometría como principal herramienta de evaluación, a la cual Pritchard (1969) la define como la aplicación de métodos matemáticos y estadísticos que ayudan a definir los procesos de la comunicación escrita, así como la naturaleza y facetas de ella, y del mismo modo a los autores que la producen. Para facilitar el análi- sis bibliométrico varios autores han establecido distintos índices bibliométricos, que son herramientas cuantificables para valorar los procesos científicos y proporcionan los datos de interés dentro del proceso investigativo sobre el impacto generado, en tiempo y espacio, tanto del documento científico como de las fuentes consultadas (Camps, 2008). Así también, se han establecido indicadores bibliométricos que son instrumentos para evaluar la información científica publicada tanto en su volumen, visibilidad, evolución y estructura. Con el análisis estadístico de estos indicadores se puede establecer el estado actual de la literatura mediante el número de publicaciones, el número de citas, su nivel de esparcimiento, la productividad tanto de autores como de instituciones, universidades, medios de comunicación escrita, etc. (Escorcia Otálora, 2014). De manera general, los indicadores bibliométricos se pueden dividir en dos grandes grupos: a) los indicadores cuantitativos de la actividad científica donde se analiza el número real de publicaciones y el estado de la ciencia, y b) los indicadores de impacto que mide la cantidad de citas que se obtienen de un trabajo y le dan importancia a un documento. En la tabla 2 se detallan los índices bibliométricos más utilizados en la actualidad.

Tabla 2. Índices bibliométricos más calculados

Índice

Indice de productividad

Grado de colaboración (Índice de Subramanyan)

Índice de antigüedad - obsolescencia
Se mide con el número de publicaciones por investigador, institución y grupo; se usa para la descripción de áreas temáticas (Arenas \& Romero, 2003).

Medida de colaboración científica entre varios autores y permite determinar el tamaño de los grupos de investigación (Escorcia Otálora, 2014).

Es utilizado para calcular el nivel de envejecimiento de la literatura científica, puede ser calculado mediante el factor de envejecimiento anual mediante el máximo de citas y la vida media (Escorcia Otálora, 2014).

Fórmula

Interpretación

$$
I P=\log N
$$

Donde:

IP = indicador de productividad personal

$\mathbf{N}=$ número de proyectos de titulación.

$$
C G=\frac{N_{m}}{N_{m}+N_{s}}
$$

Donde:

$\boldsymbol{N}_{\boldsymbol{m}}=$ Total de documentos con autoría múltiple

$\mathbf{N}_{\mathrm{s}}=$ Total de documentos de un solo autor

$$
U(t)=U(0) \times a^{t}
$$

Donde:

$\mathbf{U}(\mathbf{t})=$ utilidad residual

$\mathbf{U}(\mathbf{0})=$ utilidad total o inicial

$\mathbf{a}=$ factor de envejecimiento anual

$\mathbf{t}=$ edad
Grandes productores (10

o más trabajos) $I P \geq 1$

Productores intermedios (entre 2 y 9 trabajos) $0>I P<1$

Productores transitorios (un solo trabajo) $I P=0$

Valores cercanos a 0 indican fuerte componente de autoría simple, mientras que los cercanos a 1 denotan una fuerte proporción de autoría múltiple (Biblat, 2009).
Valor que indica la reducción de la utilidad con respecto a la del año anterior. 
Ley de Brooks para el cálculo de la utilidad de los documentos

Índice de Price

Índice de aislamiento
La utilidad total o inicial U

(0) según Ruiz Baños (1997) citado por (Boeris, 2010) representa el número total de citas que tiene un conjunto de documentos en un tiempo de años determinado.

La utilidad inicial va envejeciendo y se convierte en utilidad residual $\mathrm{U}(\mathrm{t})$, la cual llega a 0 con el tiempo.

Mide la proporción de citas en un documento que no tienen más de cinco años de antigüedad.

Indica la cantidad de referencias que pertenecen a las publicaciones del mismo país donde se edita la literatura y refleja el grado de aislamiento o apertura al exterior de un país.

$$
a=1-\frac{C}{R}
$$

Donde:

$\mathbf{R}=$ total de referencias

$\mathbf{C}=$ referencias del año con

mayor cantidad

Este valor tiene cifras entre 0 y 1 , siendo las aproximadas al 0 las de mayor envejecimiento (Boeris, 2010).

$$
I O=\frac{\text { Docs }-5 \text { años }}{\text { Total }_{\times 100 \%}}
$$

$I A=\frac{\text { Citas }_{\text {país }}}{\text { Total }_{\text {citas }}} \times 100 \%$
Los índices bibliométricos calculados dentro de esta investigación fueron:

a) Índice de productividad, se determinó la productividad científica por institución y por años, este índice guarda relación con el índice de institucionalidad.

b) Grado de colaboración, sirvió para determinar el tamaño del grupo de investigación en cuanto a los autores (estudiantes) de los trabajos de titulación de cada IES y la evolución que ha tenido en el tiempo.

c) Índice de obsolescencia, ayudó a establecer el nivel de envejecimiento de la información científica de los trabajos de titulación y se determinó mediante el factor de envejecimiento anual.

d) Índice de Price, se estableció el porcentaje de citas de los trabajos de titulación en el período 2013-2017, es decir, en los documentos que no tienen más de cinco años de antigüedad.

e) Índice de aislamiento, ayudó a determinar el porcentaje de citas que tienen los trabajos tanto de bibliografía nacional y extranjera. Para el cálculo de este índice se obtuvo una muestra de los trabajos de titulación mediante la aplicación de la fórmula de poblaciones finitas, dando un total de 67 documentos que fueron analizados. Para esto se elaboró una base de datos con la información del número de referencias bibliográficas de documentos nacionales y de documentos de otros países, también se anotó el país de procedencia de las referencias, el año de la referencia más antigua y la referencia más actual.

\section{Resultados y discusión}

\subsection{Descripción general de los trabajos de titula- ción de pregrado y posgrado}

Se observó que el período con mayor número de trabajos de titulación publicados fue entre el 20132017 , con el 50,90\% del total de trabajos de titulación incluidos en la base de datos (Figura 1). Se evidenció también que el 98,08\% corresponde a trabajos de titulación de pregrado. El 1,92\% perteneciente a posgrado, corresponde únicamente a trabajos de titulación de maestrías de la UTE y de la UDET. 


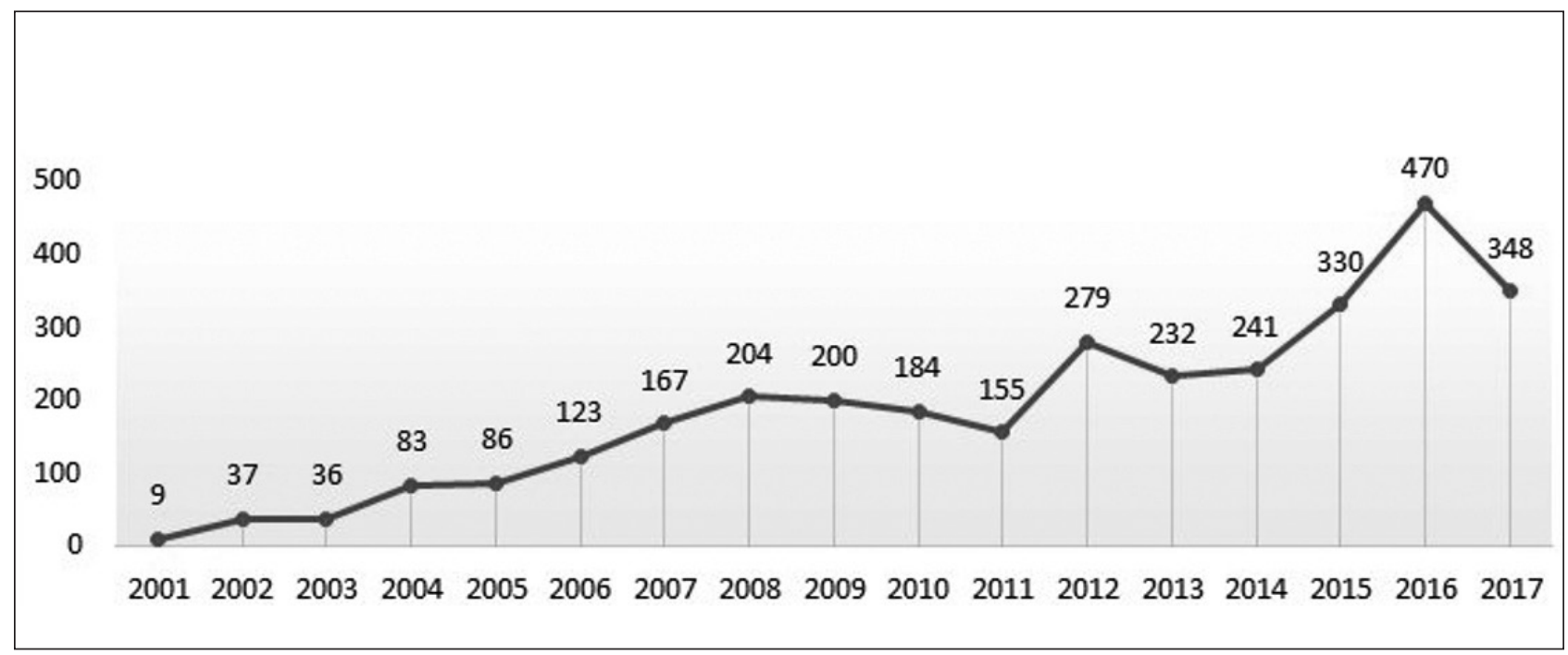

Figura 1. Evolución de los trabajos de titulación por años.

En la mayoría de las IES la rotación de docentes durante el período analizado es baja. En la UTE el número de tutores o directores es mayor, sin embargo, se han mantenido los mismos en periodos medianos y largos. En algunas de las IES se evidenció que existe alta concentración de dirección de trabajos de titulación en pocos docentes, como es el caso de USFQ y UDET. Con el promedio de los trabajos dirigidos se determinó que mientras menor es el número de trabajos de titulación, mayor es la distribución de los trabajos de titulación entre tutores o directores (Tabla 3).

Tabla 3. Tutores de trabajos de titulación de las IES

\begin{tabular}{|c|c|c|c|c|}
\hline IES & Total de tutores & $\begin{array}{c}\text { Promedio de } \\
\text { trabajos de titulación } \\
\text { dirigidos }\end{array}$ & $\begin{array}{l}\text { Rango de trabajos de } \\
\text { titulación dirigidos }\end{array}$ & $\begin{array}{l}\text { Trabajos de } \\
\text { titulación dirigidos } \\
\text { por los } 3 \text { docentes } \\
\text { más prolíficos }(\%)\end{array}$ \\
\hline UTPL & 36 & 8,42 & $1-38$ & 30,36 \\
\hline UTE & 191 & 10,66 & $1-92$ & 12,82 \\
\hline USFQ & 15 & 10,4 & $1-45$ & 68,83 \\
\hline UDET & 76 & 4,89 & $1-60$ & 47,10 \\
\hline U-ISRAEL & 28 & 4,75 & $1-19$ & 36,04 \\
\hline ESPE & 40 & 4,58 & $1-12$ & 17,49 \\
\hline
\end{tabular}

En la mayor parte de las IES predominaron los trabajos de titulación a nivel privado-local, esto responde a que la mayoría son universidades privadas cuyo enfoque es generar emprendimientos nuevos e innovadores, a excepción de la ESPE en donde la mayor parte de trabajos de titulación estuvieron enfocados al sector público. En lo referente a los trabajos de titulación dirigidos al sector privado predomi- nan los estudios de factibilidad, planes de negocio, marketing, y temáticas relacionadas, mientras que en los trabajos de titulación para el sector público se encuentran los estudios de desarrollo turístico de territorios, en donde se trató de promocionar los distintos atractivos naturales y culturales del área de estudio. En la figura 2 se observan los porcentajes de los trabajos de titulación por ámbito geográfico y por IES. 


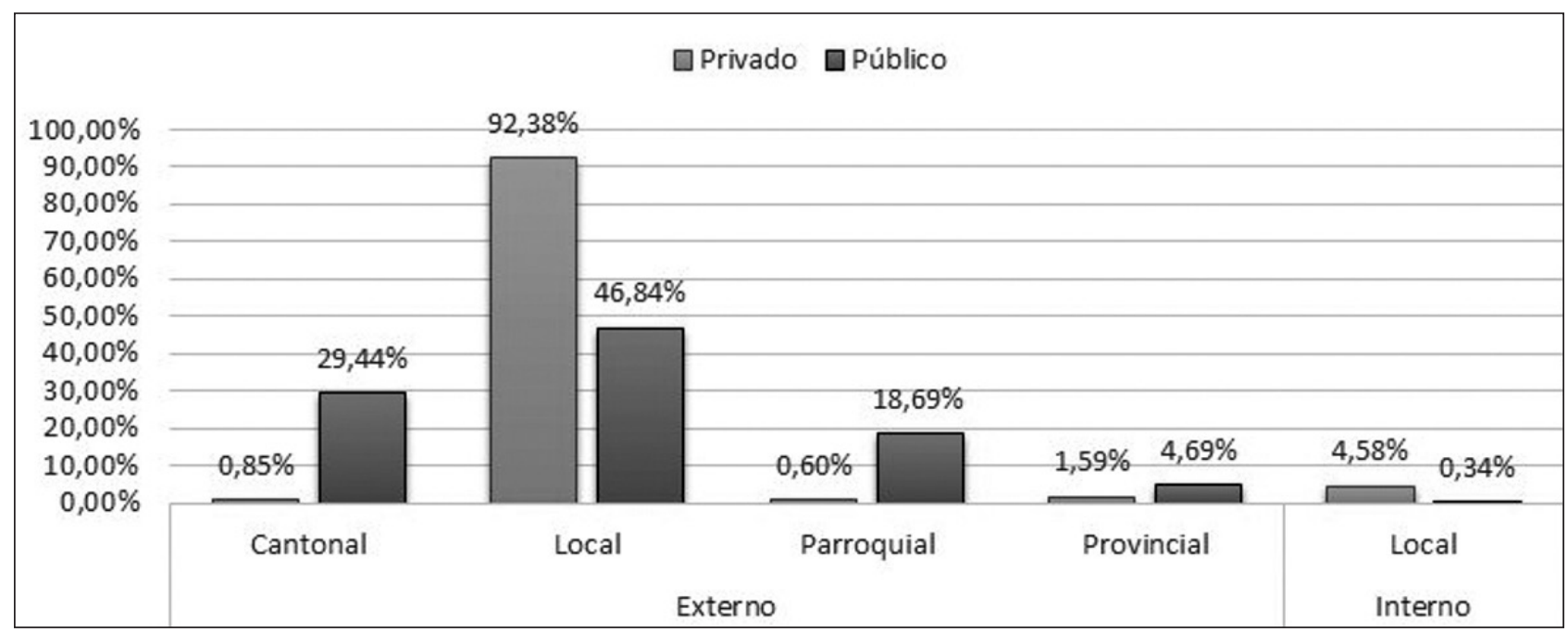

Figura 2. Tipos de proyectos publicados de acuerdo con destinatario, ámbito geográfico de aplicación y aplicación externa o interna a la IES.

\subsection{Distribución geográfica de los trabajos de titulación}

Para el análisis de los trabajos de titulación por su distribución geográfica se consideraron las nueve zonas de planificación dispuestas por la SENPLADES. Se analizaron también los datos por cantones y provincias, para determinar en cuáles de ellos se han realizado las investigaciones de los trabajos de titulación (Tabla 4). Entre las provincias con mayor número de trabajos de titulación desarrollados se encuentran: Pichincha (53,91\%), Cotopaxi (6,18\%), Imbabura (5,42\%), Loja (4,37\%), Tungurahua $(3,70 \%)$, Manabí $(3,42 \%)$ y Napo $(2,94 \%)$.

Tabla 4. Comparación de los estudios realizados por zonas de planificación

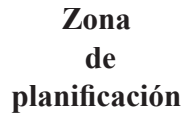

Provincias

planificación

\author{
Cantones donde se han desarrollado \\ trabajos de titulación / \\ Total de cantones
}
Parroquias donde se han desarrollado trabajos de titulación /
Total de parroquias

Esmeraldas

Imbabura

$6 / 6$

Zona 1

$$
\text { Carchi }
$$


Cotopaxi

Tungurahua

Zona 3

Zona 4

Zona 5

En la Figura 3 se observa que la mayor concentración de trabajos de titulación está en pocas parroquias, que se encuentran dentro de la zona de influencia directa de las IES (Quito). En relación con el resto de las parroquias donde se han realizado los trabajos de titulación se evidenció que en la mayoría existe entre 1 y 10 trabajos de titulación. 


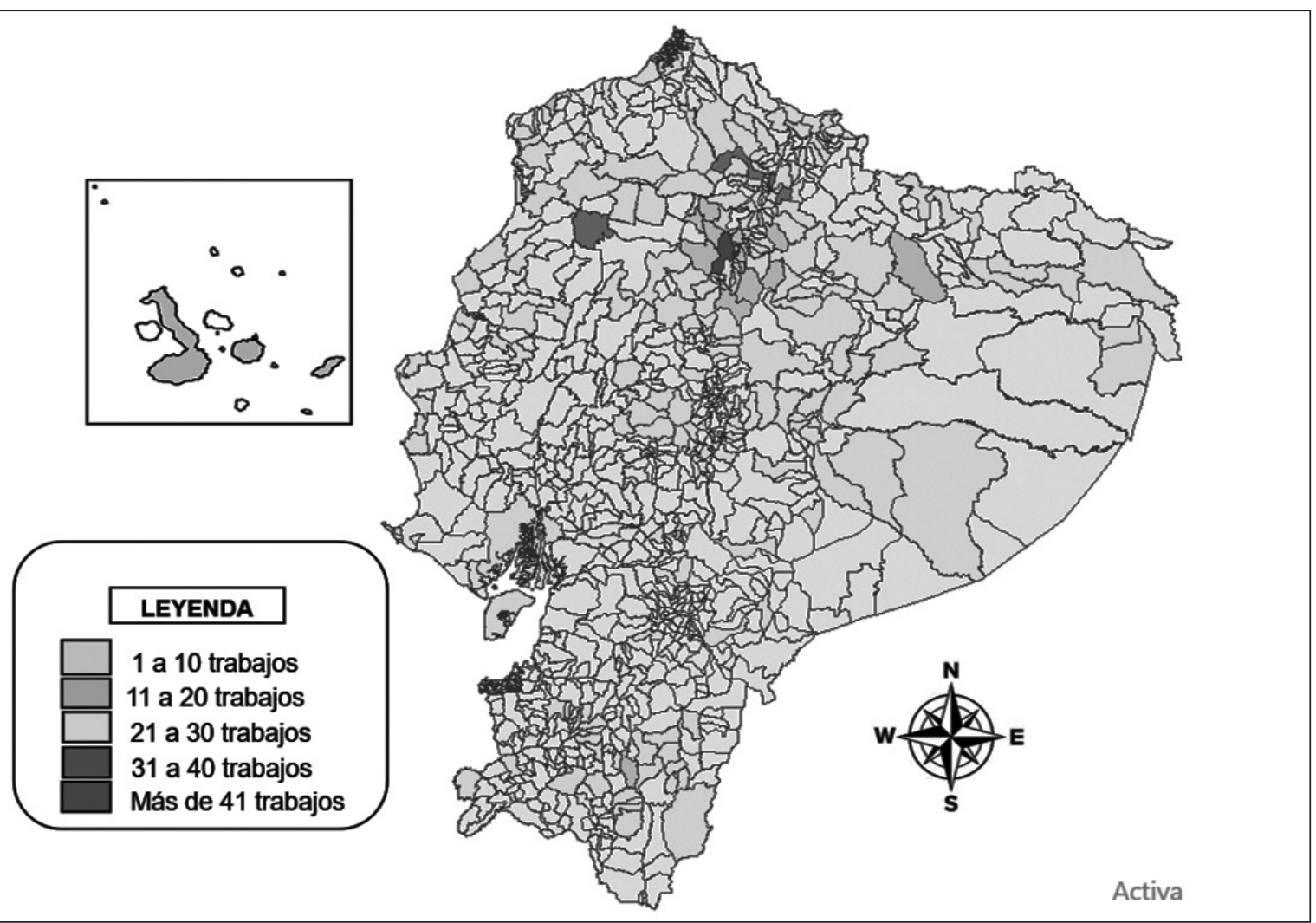

Figura 3. Mapa parroquial de concentración de trabajos de titulación.

La Zona 2 y la Zona 9, al ser el área de influencia directa de las IES tienen un alto porcentaje de trabajos de titulación desarrollados, tanto en las parroquias urbanas como en las rurales de las tres provincias que la conforman (Figura 4). La mayor concentración de trabajos de titulación se encuentra en el cantón Quito, especialmente en el Centro Histórico $(13,84 \%)$ y La Mariscal (10,45\%).

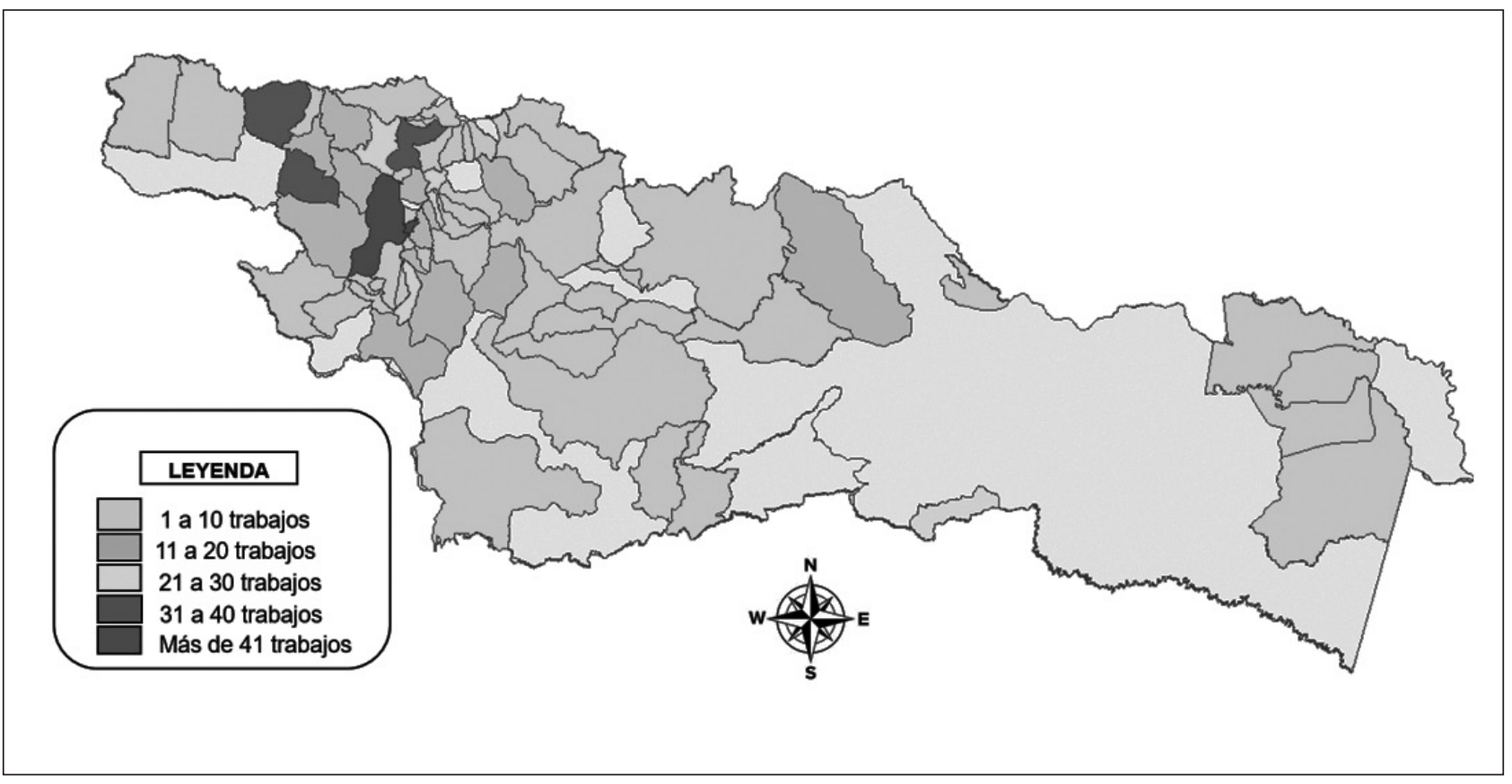

Figura 4. Mapa parroquial de concentración de trabajos de titulación de la Zona 2. 


\subsection{Líneas de investigación de los trabajos de titulación}

Dentro de las líneas de investigación consideradas, el mayor porcentaje de trabajos de titulación corresponden a la línea "Estudios económicos y administrativos" $(62,20 \%)$, en la línea "Turismo alternativo" se obtuvo el 17,93\%, en "Territorio" el 12,97\%, y en "Patrimonio turístico" el 4,14\% (Figura 5). Cabe mencionar que algunos de los trabajos tuvieron temáticas muy variadas y diferentes a las de las líneas de investigación consideradas, a ese grupo se le agrupó en "Otros", representando el 2,76\%. Se identificaron temáticas que no han sido objeto de investigación, entre ellas la relación entre turismo, TICs y aspectos digitales, las nuevas tendencias de los turistas en Ecuador, el turismo en el cambio de la matriz productiva y las perspectivas del turismo a futuro.

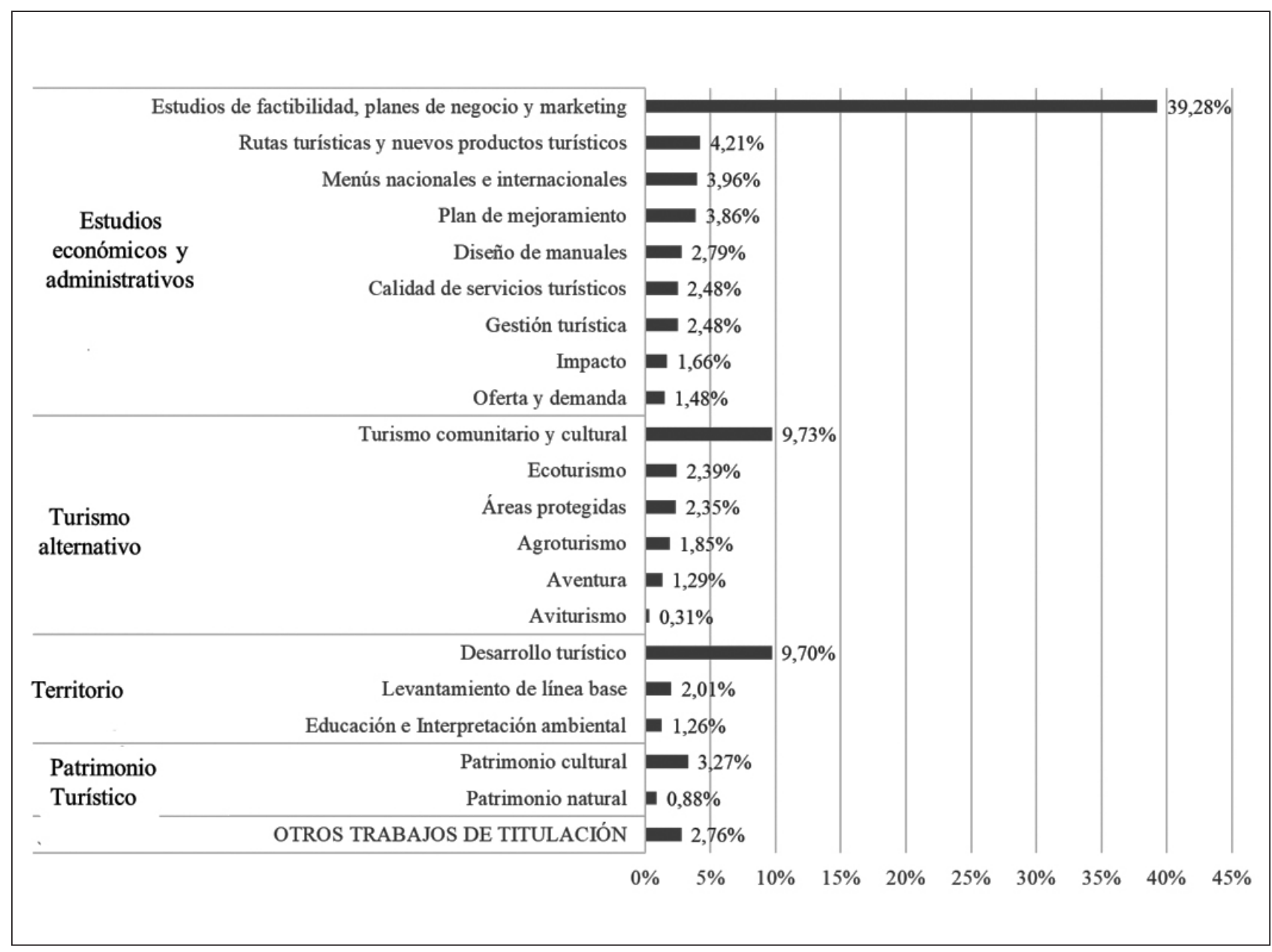

Figura 5. Líneas de investigación y temas de los trabajos de titulación.

\subsection{Productividad y colaboración en los trabajos de titulación}

El cálculo del índice de productividad dio como resultado que las seis IES son grandes productoras, ya que los índices son mayores que 1. La UTE es la de mayor productividad, resultado esperado dado el alto número de estudiantes titulados por la antigüedad de sus carreras (Figura 6).

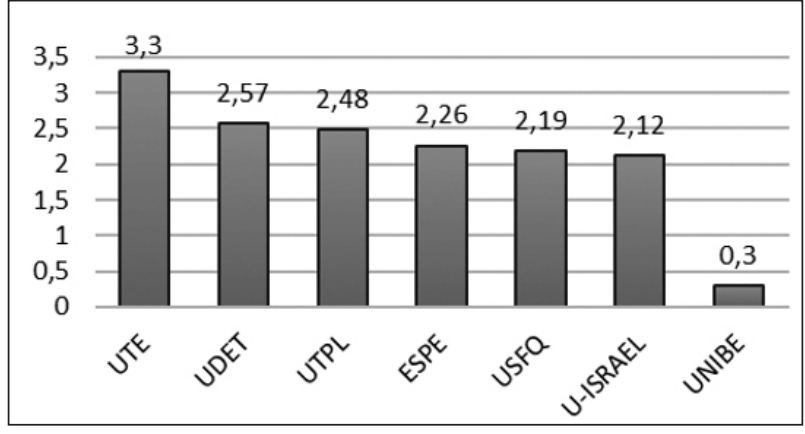

Figura 6. Índice de productividad por IES. 
En la progresión de la Figura 7 se observa que en el año 2001 la producción fue intermedia con un índice de 0,95 , mientras que con el paso de los años los trabajos de titulación aumentaron. El año con mayor producción fue el 2016, con un total de 470 trabajos de titulación que representan un índice del 2,67.

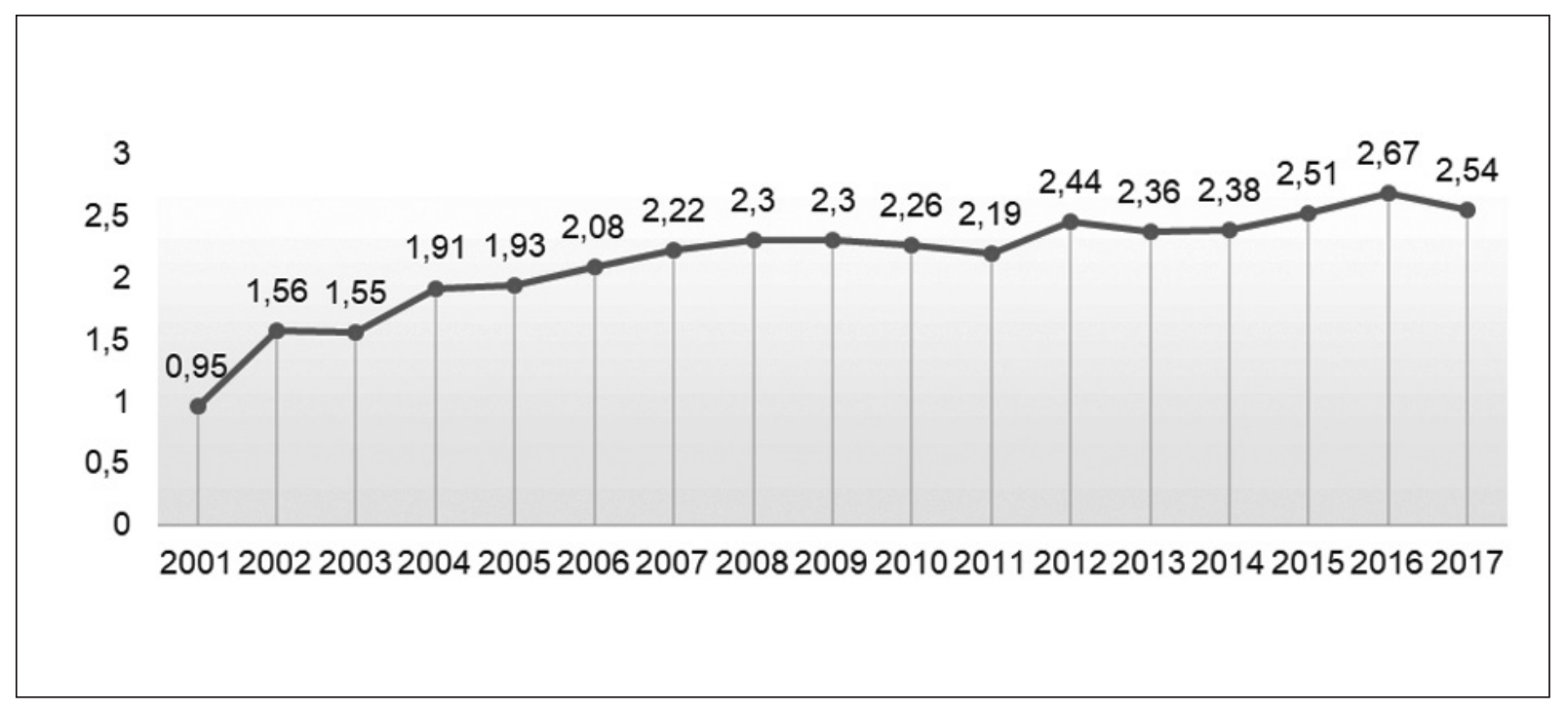

Figura 7. Índice de productividad por años.

Para el cálculo del grado de colaboración se consideraron los trabajos de titulación individuales de cada IES y los trabajos de titulación con múltiples autores, observándose que la mayoría de trabajos de titulación corresponden al primer caso. En el caso de múltiples autores, el índice es mayor en la ESPE (Fi- gura 8), IES que presenta un grado de colaboración medio, mientras que en la UTE, con la mayor población estudiantil, el grado de colaboración es prácticamente nulo. En cuestión del tamaño del grupo de investigación, la USFQ y la UDET tuvieron trabajos de titulación de hasta cuatro estudiantes.

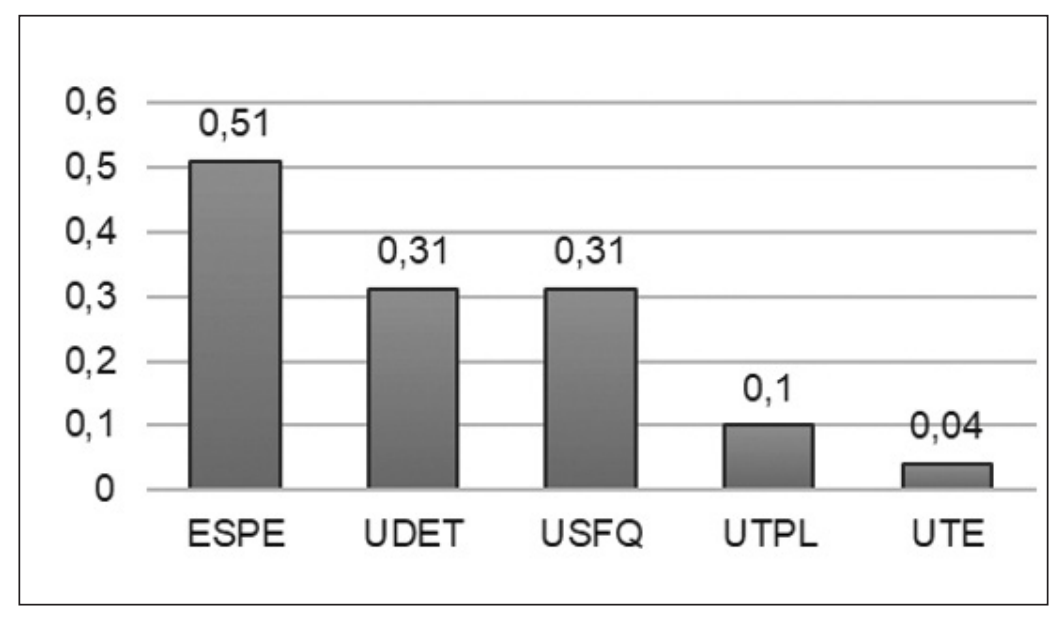

Figura 8. Grado de colaboración por IES.

El grado de colaboración durante el período analizado ha cambiado notablemente a partir del 2004, año en el que se realizaron mayores colaboraciones en las investigaciones con un índice de 0,58 (los valores más cercanos a uno indican que hubo mayor número de trabajos de titulación con múltiples autores), a partir de ese punto el tamaño de los grupos de investigación y las colaboraciones bajaron significativamente y los valores se mantienen (Figura 9). 


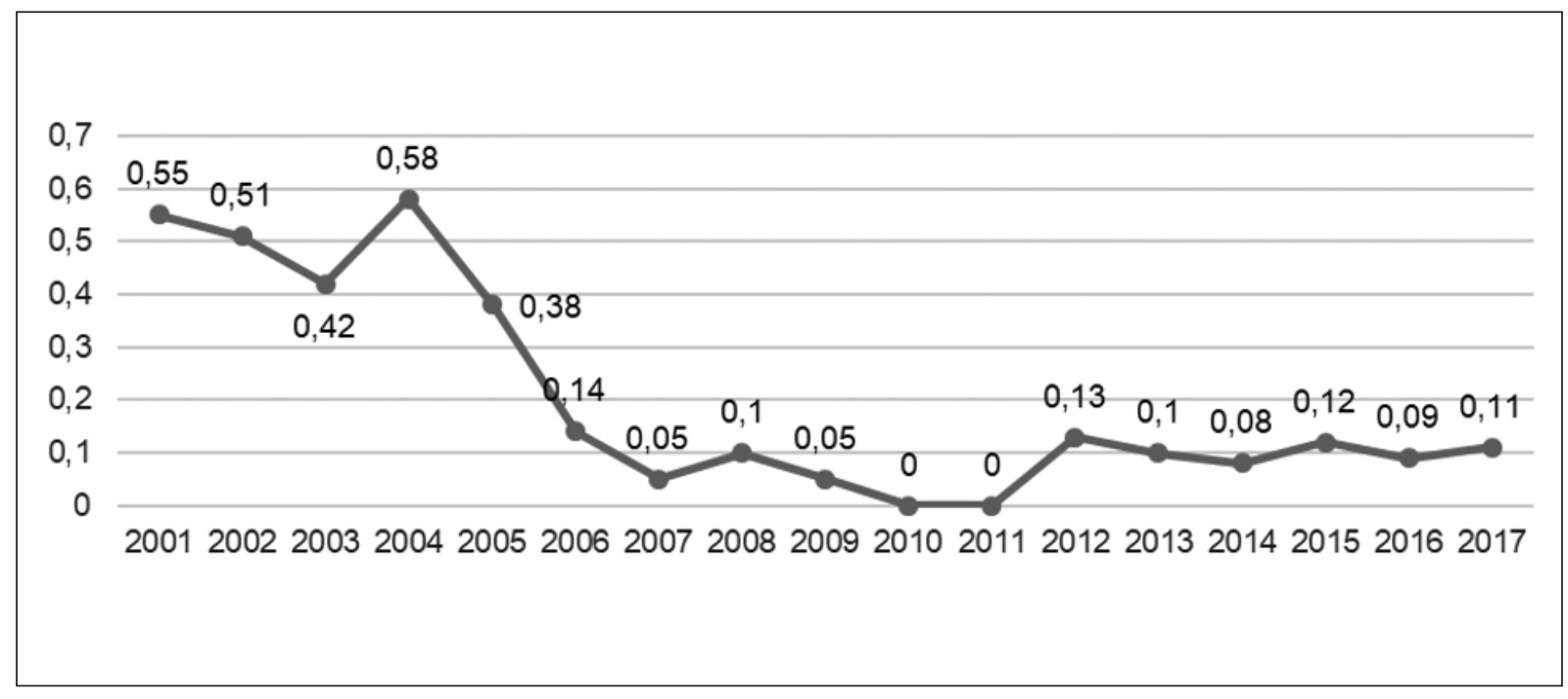

Figura 9. Grado de colaboración por años.

\subsection{Análisis de bibliografia citada}

En la tabla 5 se recoge la sumatoria absoluta de las referencias incluidas en todos los trabajos de titulación incluidos en el estudio, siendo la UTE quien posee mayor número, coherente con ser la IES con mayor número de trabajos de titulación desarrolla- dos durante el período evaluado. Se observó que en la mayoría de las IES que fueron parte de esta investigación, las fuentes de consulta más utilizadas provienen de páginas web, con excepción de la UTE que tienen un valor superior de consultas realizadas en libros físicos, en especial en las carreras de posgrado, en donde la revisión bibliográfica fue extensa.

\begin{tabular}{ccc} 
IES & $\begin{array}{c}\text { Documentos } \\
\text { científico-técnicos }\end{array}$ & Páginas web \\
\hline UTE & $22.129(56,97 \%)$ & $16.717(43,03 \%)$ \\
U-ISRAEL & $1.562(49,84 \%)$ & $1.572(50,16 \%)$ \\
ESPE & $1.942(42,13 \%)$ & $2.668(57,87 \%)$ \\
UTPL & $2.917(40,28 \%)$ & $4.324(59,72 \%)$ \\
UDET & $2.730(38,21 \%)$ & $4.414(61,19 \%)$ \\
USFQ & $642(28,34 \%)$ & $1.623(71,66 \%)$ \\
\hline
\end{tabular}

Tabla 5. Número de referencias en los trabajos de titulación por IES

Igualmente, con el paso de los años se evidencia que la revisión de bibliografía aumenta significativamente en número, y en cuanto a calidad, se entendería que también se ha incrementado por el tipo de documentos de consulta, pero se requiere profundizar en el análisis e incluir diferentes puntos de vista de los autores. En la figura 10 se muestra el aumento de referencias realizadas a partir del año 2010, hay que mencionar también que los archivos de los trabajos de titulación anteriores a este año no están disponibles, por lo que no se pudo recopilar esa información de todos los trabajos de titulación. En la tabla 6 se detalla el promedio de referencias en los trabajos de titulación por año de publicación. 


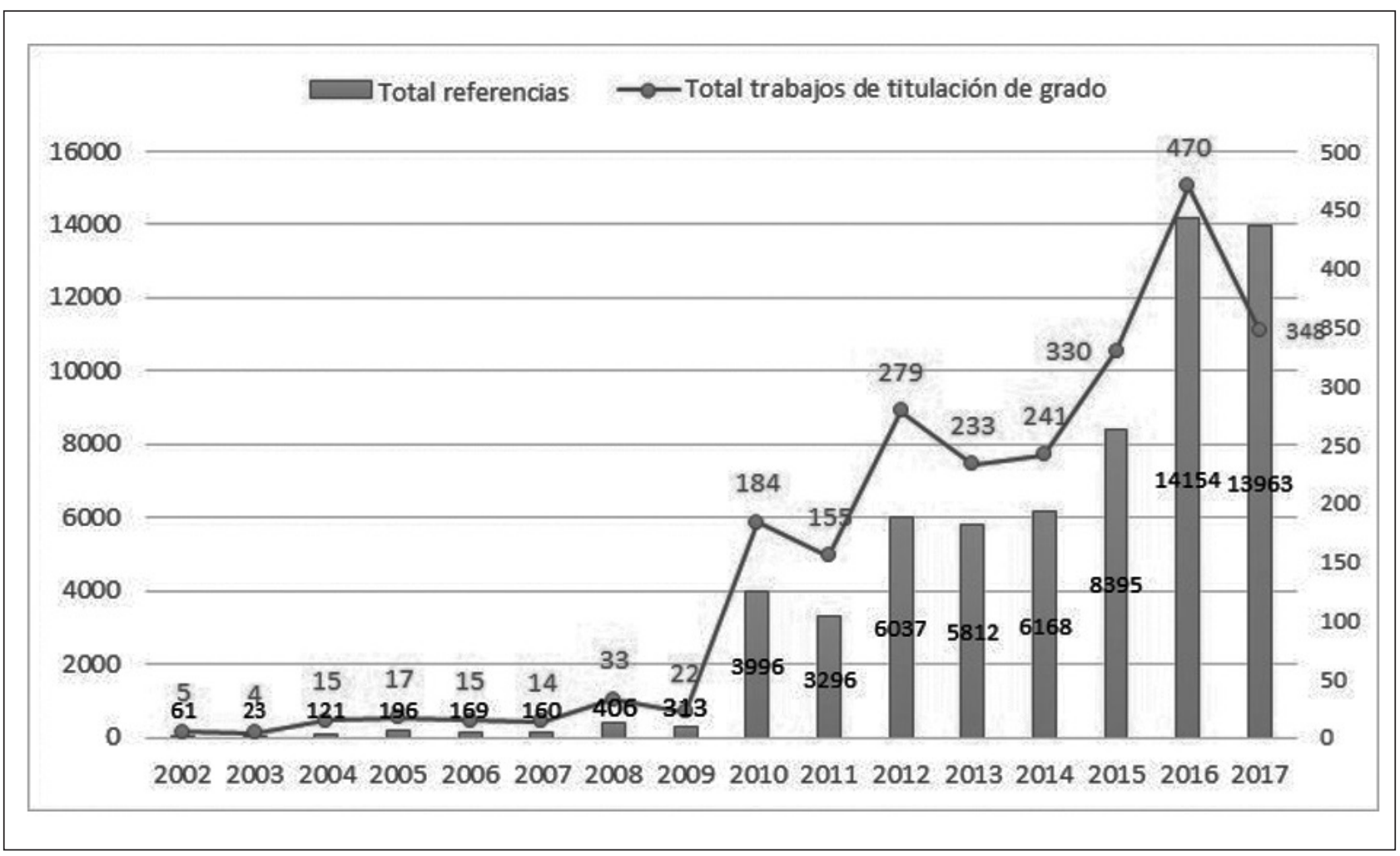

Figura 10. Evolución de las referencias bibliográficas en los trabajos de titulación.

Tabla 6. Promedio de referencias bibliográficas por año por trabajo de titulación

\begin{tabular}{ccccccccc} 
Año & $\mathbf{2 0 0 2}$ & $\mathbf{2 0 0 3}$ & $\mathbf{2 0 0 4}$ & $\mathbf{2 0 0 5}$ & $\mathbf{2 0 0 6}$ & $\mathbf{2 0 0 7}$ & $\mathbf{2 0 0 8}$ & $\mathbf{2 0 0 9}$ \\
\hline Promedio & 12,5 & 5,75 & 8,07 & 11,53 & 11,27 & 11,43 & 12,3 & 14,23 \\
\hline Año & $\mathbf{2 0 1 0}$ & $\mathbf{2 0 1 1}$ & $\mathbf{2 0 1 2}$ & $\mathbf{2 0 1 3}$ & $\mathbf{2 0 1 4}$ & $\mathbf{2 0 1 5}$ & $\mathbf{2 0 1 6}$ & $\mathbf{2 0 1 7}$ \\
\hline Promedio & 21,72 & 21,26 & 21,64 & 24,94 & 25,59 & 26,05 & 30,11 & 40,12 \\
\hline
\end{tabular}

\section{6. Índice de obsolescencia}

Al realizar los cálculos del factor de envejecimiento anual mediante el máximo de citas se obtuvo un factor de 0,78 . Con este dato se determinó que la pérdida de utilidad por año de los proyectos de titulación analizados es del $22 \%$, porque correspondería a documentos de más de 10 años de antigüedad que podrían considerarse desactualizados, con excepción de documentos cuya información se mantiene vigente con el paso de los años. De modo que cada año la literatura queda reducida un
$78 \%$ con respecto al año anterior. Dado el paso de los años, varias investigaciones pueden perder validez por el dinamismo de la actividad turística

Para determinar la antigüedad de las citas de los trabajos de titulación se comparó la referencia más antigua y la más reciente, en relación con el año de publicación de los trabajos de titulación que fueron parte de la muestra. Se observó que existe literatura citada muy obsoleta (Figura 11) que corresponde a libros y revistas, la referencia más antigua que se encontró fue de 47 años de antigüedad. 


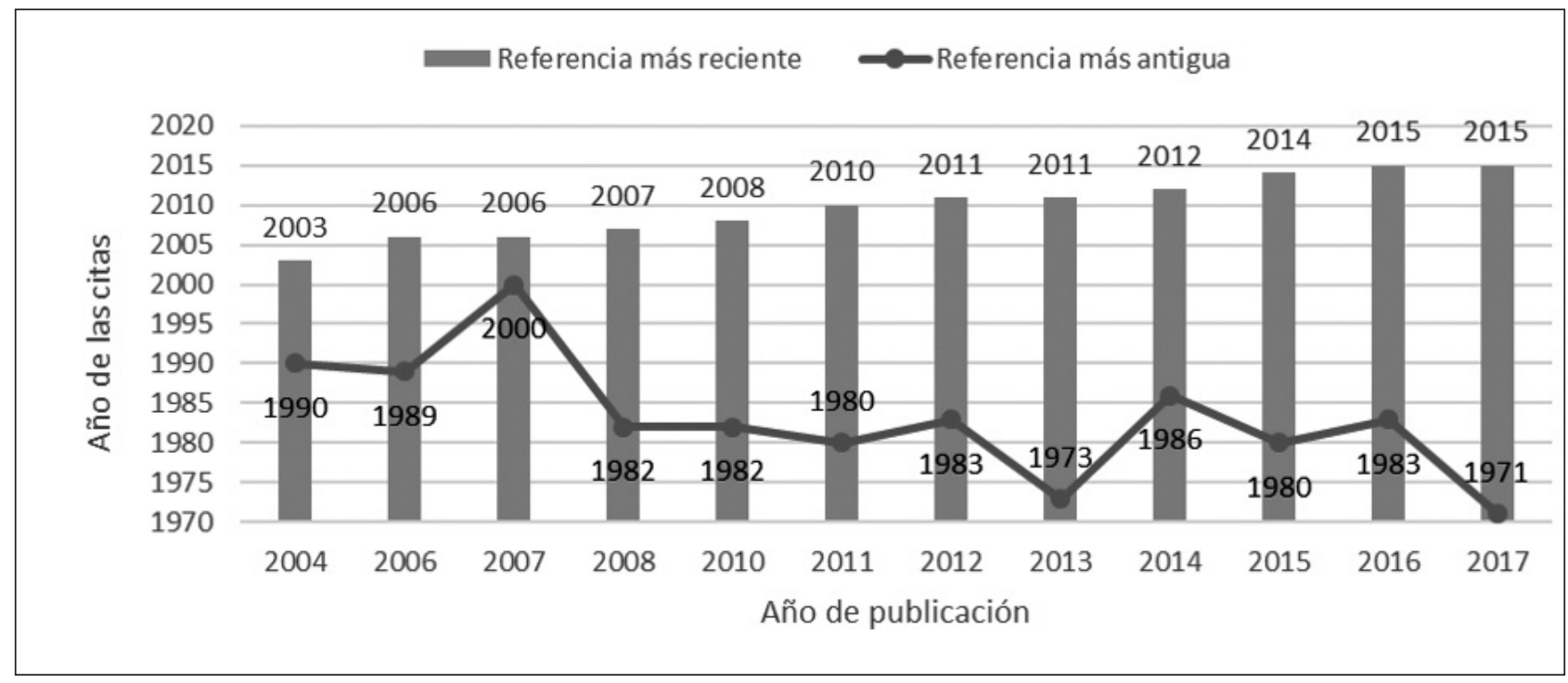

Figura 11. Comparación entre el año de las referencias y el año de publicación de los trabajos de titulación.

Con el índice de Price se determinó el porcentaje de antigüedad de las citas en los trabajos de titulación de cinco años atrás de cada IES. La institución que obtuvo el mayor índice fue la UTE $(14,49)$ debido a que tiene más publicaciones en comparación con las demás instituciones, que tienen un índice bajo, como es el caso de UTPL $(3,24)$, UDET $(2,74)$, U-ISRAEL $(2,02)$ y USFQ $(0,87)$. Según Price (1986) citado por Escorcia (2008), este índice varía dependiendo del área de conocimiento, pues- to que en ciencias duras la información se mantiene con el paso de los años, a diferencia de las ciencias blandas que son dinámicas y cambiantes, como es el caso del turismo.

Los valores obtenidos por el cálculo del índice de aislamiento muestran que existe amplia apertura a bibliografía extranjera, debido a que se obtuvieron altos valores en el período analizado, con excepción del 2008 que el valor fue bajo y tuvieron mayor peso los documentos científico-técnicos nacionales (Figura 12).

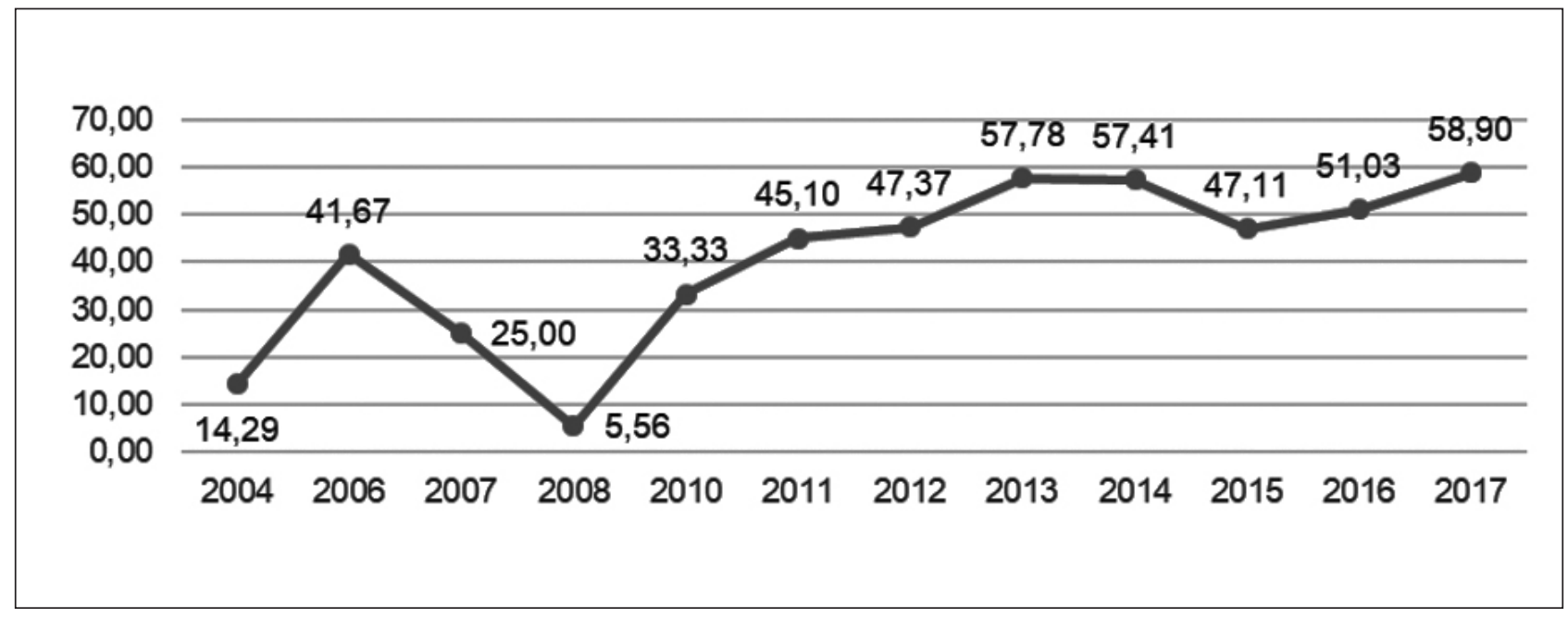

Figura 12. Índice de aislamiento por años.

En cuanto a los países más citados, destacan México y España, como se detalla en la tabla 7, cuyas publicaciones citadas corresponden a un número importante de libros que son reconocidos en el ámbito turístico, y que contienen información conceptual para los trabajos de titulación. 
Tabla 7. Número de referencias por país

\begin{tabular}{lcc}
\multicolumn{1}{c}{ País } & $\mathbf{N}^{\circ}$ de citas & $\%$ \\
\hline México & 168 & $37,25 \%$ \\
España & 148 & $32,82 \%$ \\
Colombia & 43 & $9,53 \%$ \\
Italia & 18 & $3,99 \%$ \\
Argentina & 14 & $3,10 \%$ \\
Estados Unidos & 13 & $2,88 \%$ \\
Otros & 47 & $10,42 \%$ \\
\hline Total general & 451 & $\mathbf{1 0 0 , 0 0 \%}$ \\
\hline
\end{tabular}

\section{Conclusiones}

Los trabajos de titulación publicados durante el período 2000-2017 de las instituciones de educación superior incluidas en esta investigación es alta, puesto que se recopilaron 3.185 trabajos de titulación, tanto de pregrado $(98,08 \%)$ como de posgrado. En cuanto a la distribución geográfica de los trabajos de titulación, se observó una concentración en el área de influencia directa de las IES consideradas (Pichincha, seguido de Cotopaxi e Imbabura), y que, a mayor distancia, menor número de trabajos de titulación. El cantón con mayor porcentaje de trabajos de titulación realizados es Quito, especialmente en el Centro Histórico y La Mariscal.

De las líneas de investigación consideradas en el análisis, el $62,20 \%$ de los trabajos de titulación corresponden con la línea "Estudios económicos y administrativos", predominando la elaboración de estudios de factibilidad y planes de negocios para empresas privadas. Es decir, se ha primado el trabajo con la empresa privada en el desarrollo de nuevos emprendimientos. Las líneas de investigación "Turismo alternativo" y "Territorio" representaron la tercera parte de los trabajos de titulación, principalmente direccionados hacia el turismo comunitario y cultural, y los planes de desarrollo turístico, respectivamente.

Los valores obtenidos a través de los índices bibliométricos señalan a la UTE como la IES con mayor producción científica, valores acordes con su oferta académica amplia y su antigüedad. La ESPE presenta el mayor nivel de colaboración entre docentes en la dirección de trabajos de titulación. La UDET y la USFQ se caracterizan por los grupos de investigación más grandes, con múltiples autores y publicaciones de hasta cuatro investigadores. La literatura publicada tiene un envejecimiento alto, debido a que el turismo es multidisciplinar, en donde los conocimientos relacionados avanzan rápido por su fuerte vínculo con la sociedad, la tecnología y la aparición de nuevas tendencias. Por último, se destaca el uso de literatura extranjera, principalmente de México y España, así como el uso de literatura con una antigüedad superior a 2008, y que presenta una alta similitud en las fuentes consultadas. 


\section{Referencias}

Amat Noguera, N. (1994). La documentación y sus tecnologías. Madrid: Pirámide.

Arenas, S., \& Romero, A. (2003). Indicadores bibliométricos de la revista científica Zootecnia Tropical. Zootecnia Tropical, 21(3), 325-350.

Asamblea Nacional del Ecuador. Código Orgánico de la Economía Social del Conocimiento, la Creatividad y la Innovación (2016). Registro Oficial No 899.

Azevedo, J., Faria, S., Gomes, C. L., Federal, U., \& Gerais, D. M. (2013). El ocio y el turismo en los artículos publicados en revistas académicas de turismo. Estudios y Perspectivas en Turismo, 22, 875-892.

Barrios, M., Borrego, A., Vilaginés, A., Ollé, C., \& Somoza, M. (2008). A bibliometric study of psychological research on tourism. Scientometrics, 77(3), 453-467. https://doi.org/10.1007/ s11192-007-1952-0

Bienert, I. R. C., Oliveira, R. C. de, Andrade, P. B. de, \& Caramori, C. A. (2015). Bibliometric indexes, databases and impact factors in cardiology. Revista Brasileira de Cirurgia Cardiovascular, 30(2), 254-9. https://doi. org/10.5935/1678-9741.20150019

Boeris, C. E. (2010). Aplicación de métodos bibliométricos a la evaluación de colecciones: el caso de la Biblioteca del Instituto Argentino de Radioastronomía. Universidad Nacional de La Plata.

Breilh, J. (2015). Reflexiones sobre las potencialidades y límites de los índices de cienciometría (bibliometría científica) convencionales. In Academia Ecuatoriana de Medicina. Universidad Andina Simón Bolívar.

Broadus, R. N. (1987). Toward a definition of "bibliometrics." Scientometrics, 12(5-6), 373-379. https://doi.org/10.1007/BF02016680

Camps, D. (2008). Limitaciones de los indicadores bibliométricos en la evaluación de la actividad científica biomédica. Colombia Médica, 39(1), 74-79. https://doi.org/10.1016/j. mseb.2004.03.008
Cheng, M., Edwards, D., Darcy, S., \& Redfern, K. (2018). A Tri-Method Approach to a Review of Adventure Tourism Literature: Bibliometric Analysis, Content Analysis, and a Quantitative Systematic Literature Review. Journal of Hospitality \& Tourism Research, 42(6), 997-1020. https://doi.org/10.1177/1096348016640588

Consejo de Educación Superior (CES). Reglamento de Régimen Académico (2017). Ecuador.

Díaz Macías, T. M., \& Rodríguez Cevallos, M. (2017). Indicadores bibliométricos de las tesis de pregrado en el repositorio digital de la Facultad de Ciencias Humanísticas y Sociales de la Universidad Técnica de Manabí, Ecuador, periodo 2015-2016. ReHuSo: Revista de Ciencias Humanísticas y Sociales, 2(1), 102-116.

Durán-Sánchez, A., Álvarez-García, J., \& Del Río-Rama, M. de la C. (2015). La investigación en turismo activo: revisión bibliográfica (1975-2013). ROTUR: Revista de Ocio y Turismo, 8(1), 62-76. https://doi.org/10.17979/ rotur.2015.8.1.1296

Durán-Sánchez, A., Del Río-Rama, M. de la C., \& Álvarez-García, J. (2017). Bibliometric analysis of publications on wine tourism in the databases Scopus and WoS. European Research on Management and Business Economics, 23(1), 8-15. https://doi.org/10.1016/J.IEDEEN.2016.02.001

Ellegaard, O., \& Wallin, J. A. (2015). The bibliometric analysis of scholarly production: How great is the impact? Scientometrics, 105(3), 1809-1831. https://doi.org/10.1007/s11192-015-1645-z

Escorcia Otálora, T. A. (2014). El análisis bibliométrico como herramienta para el seguimiento de publicaciones cientificas, tesis y trabajos de grado. Pontificia Universidad Javeriana.

Estevão, C., Garcia, A. R., \& Fernandes, C. (2017). Convergence in tourism management research : a bibliometric analysis, 13(4), 30-42. https:// doi.org/10.18089/tms.2017.13404

Evren, S., \& Kozak, N. (2014). Bibliometric analysis of tourism and hospitality related articles publi- 
shed in Turkey. Anatolia, 25(1), 61-80. https:// doi.org/10.1080/13032917.2013.824906

Ferreiro Aláez, L. (1993). Bibliometría: (análisis bivariante). Madrid: Eypasa.

Garrigos-Simon, F., Narangajavana-Kaosiri, Y., Lengua-Lengua, I., Garrigos-Simon, F. J., Narangajavana-Kaosiri, Y., \& Lengua-Lengua, I. (2018). Tourism and Sustainability: A Bibliometric and Visualization Analysis. Sustainability, 10(6), 1976. https://doi.org/10.3390/ su10061976

González de Dios, J., Moya Benavent, M., \& Mateos Hernández, M. A. (1997). Indicadores bibliométricos: características y limitaciones en el análisis de la actividad científica. Anales españoles de pediatría: Publicación Oficial de la Asociación Española de Pediatría ( AEP), 47(3), 235-244.

Güzeller, C. O., \& Çeliker, N. (2018). Bibliometric Analysis of Tourism Research for the Period 2007-2016. Advances in Hospitality and Tourism Research (AHTR), 6(1), 1-22. https://doi. org/10.30519/ahtr.446248

Moragues Cortada, D. (2006). Turismo, cultura $y$ desarrollo. Madrid: Agencia Española de Cooperación Internacional para el Desarrollo. Obtenido de: http://data.cervantesvirtual.com/manifestation/251940? $\mathrm{ga}=2.207001109 .72969750 .1542412203$ 716502524.1542412203

Morocho Guerra, J. G. (2018). Análisis bibliométrico de la producción científica en el sistema de educación superior ecuatoriano. Escuela Politécnica Nacional. Retrieved from http://bibdigital.epn.edu.ec/handle/15000/19202

Palomo, J., Figueroa-Domecq, C., Flecha-Barrio, D., \& Segovia-Pérez, M. (2017). The Use of New Data Analysis Techniques in Tourism: A Bibliometric Analysis in Data Mining, Big Data and Structural Equations Models. E-Review of Tourism Research (ERTR), 8 .

Peralta González, M. J., Frías Guzmán, M., \& Chaviano, O. G. (2015). Criterios, clasificaciones y tendencias de los indicadores bibliométricos en la evaluación de la ciencia. Revista Cubana de Información en Ciencias de la Salud,
26(3), 290-309. https://doi.org/10.1016/j. cnur.2005.07.008

Pineda Ospina, D. L. (2015). Análisis bibliométrico para la identificación de factores de innovación en la industria alimenticia. Revista Ad-Minister, (27), 95-126.

Pritchard, A. (1969). Statistical Bibliography or Bibliometrics? Journal of Documentation, 25(4), 348-349.

Rueda-Clausen Gómez, C. F., Villa-Roel Gutíerrez, C., \& Rueda-Clausen Pinzón, C. E. . (2010). Indicadores bibliométricos: origen, aplicación, contradicción y nuevas propuestas. Med UNAB, 8(1), 29-36. https://doi. org/10.29375/01237047.208

Ruhanen, L., Weiler, B., Moyle, B. D., \& Mclennan, J. (2015). Trends and patterns in sustainable tourism research: a 25 -year bibliometric analysis. Journal of Sustainable Tourism, 23(4), 517-535. https://doi.org/10.1080/09669582.2014.978790

Satyanarayana, D. (2014). TOURISMOS: A Bibliometric Study. Journal of Tourism \& Hospitality, 04(02), 1-3. https://doi.org/10.4172/21670269.1000124

Sisa Caiza, I. F. (2010). Análisis bibliométrico de la producción científica en ciencias de la salud en Ecuador 1999-2009: Hacia una política de investigación y desarrollo en función de las prioridades de salud del país. Universidad San Francisco de Quito.

Solano López, E., Castellanos Quintero, S. J., López Rodríguez del Rey, M. M., \& Hernández Fernández, J. I. (2009). La bibliometría: una herramienta eficaz para evaluar la actividad científica postgraduada. Revista Científica de las Ciencias Médicas en Cienfuegos, 7(4), 59-62.

Tintoré Subirana, J., Diedrich, A., Vizoso, G., \& Pitarch, S. (2007). Gestión integrada de las zonas costeras basada en el conocimiento: un elemento clave para garantizar un turismo sostenible. Estudios Turísticos, 172/173, 213-217.

Torrijo Quindemil, E. M., \& Cedeño Espinoza, R. D. (2017). Análisis bibliométrico de la literatura científica publicada en la revista La Técnica (2010-2016). ReHuSo: Revista de Ciencias Humanísticas y Sociales, 2(1), 1-20. 